\title{
STABILITY OF COLUMNS SUBJECTED TO PERIODIC AXIAL FORCES OF IMPULSIVE TYPE*
}

\author{
$\mathrm{BY}$ \\ NORMAN J. FINIZIO \\ University of Rhode Island
}

1. Introduction. Our aim here is to present an analysis of the motion of columns with pinned ends subjected to periodic axial forces of impulsive type. We are primarily concerned with the determination of whether or not the motion of the column is stable, where by a stable motion we mean that the transverse oscillations of the column are bounded for all $t>0$ when arbitrary initial conditions are prescribed. The study of transverse oscillations of columns is a relatively old problem in mechanics [13]; nevertheless, it is a problem of current interest [9], [10]. The new aspect presented here is embodied in the type of axial force assumed and in the analysis of the resulting motion. We have assumed that the periodic axial force $F$ is of the form $F(t)=\mu+\eta \delta_{p}(t)$, where $\mu$ and $\eta$ are real parameters, $t$ is the time variable, and $\delta_{p}(t)=\sum_{-\infty}^{\infty}(-1)^{n} \delta(t-n p / 2)$, with $\delta(t)$ denoting the Dirac $\delta$-function and $p>0$ a constant. Lubkin and Stoker [5], in 1943, investigated columns subjected to forces of the form $F(t)=\mu+\eta \cos (2 \pi / p) t$. This paper of Lubkin and Stoker has motivated the present investigation.

The focal point of the analysis is the set of second-order linear ordinary differential equations $\left(\beta_{n}, \alpha_{n}\right.$, and $d(>0)$ denote real parameters)

$$
y_{n}^{\prime \prime}(t)+2 d y_{n}^{\prime}(t)+\left[\beta_{n}+\alpha_{n} \delta_{2 \pi}(t)\right] y_{n}(t)=0, \quad n=1,2,3 \cdots,
$$

since the decision as to the stability of the column depends on the character of the set of all solutions of these equations. An added feature of Eqs. (1.1) is that they offer an example of Hill's equation for which many of the ramifications of the theory of such equations can be exhibited explicitly (such examples are rare indeed). For a rather complete and extensive treatment in this direction see [2].

2. General description of the problem. For the analytical description of the transverse oscillations of columns we make use of the standard theory, as developed, for example, in [14]. Further, we assume that the column is subjected to axial forces $F$ depending on the time $t$ and applied at the ends of the column (these forces are taken to be positive when they are tensions) and a lateral damping force that is proportional to the velocity of the lateral deflection. We denote by $f(t, x)$ the forces in the column

* Received June 28, 1972; revised version received October 24, 1972. This material comprises a portion of the author's doctoral dissertation at the Courant Insitute of Mathematical Sciences, New York University. The author wishes to acknowledge his deep sense of gratitude to Professor James J. Stoker of the Courant Institute for suggesting this problem and for his patient and considerate guidance during its development. 
induced by the application of the forces $F$ at its ends; here $x$ represents the distance of a cross-section of the column from a fixed origin (usually the midpoint of the column or one of its ends). With these assumptions the partial differential equation for the lateral deflection $w(x, t)$ of the column becomes [14, p. 374]

$$
E I \frac{\partial^{4} w}{\partial x^{4}}-\frac{\partial^{2}}{\partial x^{2}}\{f(t, x) w\}+h \frac{\partial w}{\partial t}+m \frac{\partial^{2} w}{\partial t^{2}}=0 .
$$

In this equation the flexural rigidity, $E I$, is the product, respectively, of the Young's modulus of the column and the moment of inertia of its cross-section, $m$ denotes the mass per unit length, and $h(>0)$ is the damping constant.

Throughout we assume that $F$ is given by

$$
F(t)=\mu+\eta \delta_{p}(t)=\mu+\eta \sum_{-\infty}^{\infty}(-1)^{n} \delta\left(t-\frac{n p}{2}\right) ;
$$

i.e., $F$ consists of a steady portion $\mu$ and a periodic portion which is impulsive in nature $(\mu, \eta$, and $p(>0)$ are real parameters). $\delta(t)$ denotes, of course, the Dirac delta function. With the periodic portion of $F$ we can associate an "amplitude" $\eta$ and a frequency $\nu=1 / p$.

In the paper of Lubkin and Stoker [5], it is argued that for most practical situations $f(t, x)$ may be assumed to be essentially the same as $F(t)$, since the longitudinal oscillations of the column have very large frequencies as compared with the transverse oscillations. The same assumption is made here. For convenience we introduce in Eq. (2.1) new dimensionless independent variables $\tau=2 \pi \nu t$ and $\xi=\pi x / l$, where $l$ represents the length of the column and $\nu=1 / p$. The minimum load beyond which the column buckles is known as the Euler load, denoted by $-P_{E}$, with $P_{E}=E I \pi^{2} / l^{2}[14$, p. 375]. We denote by $\rho$ the density of the column and also introduce the notation $\epsilon_{0} \equiv P_{E} / E A$, where $A$ is the cross-sectional area of the column; thus $\epsilon_{0}$ is the initial uniform compressive longitudinal strain. Finally, we note that the fundamental frequency of the free longitudinal vibration of a column having a single node at its center is denoted by $\nu_{0}$ and is given by [5]

$$
\nu_{0}=\frac{1}{2 l}(E / \rho)^{1 / 2} .
$$

With this notation Eq. (2.1) has the form $(d=h / 4 \pi m \nu)$

$$
\frac{\partial^{4} w}{\partial \xi^{4}}-\frac{1}{P_{E}} F\left(\frac{p}{2 \pi} \tau\right) \frac{\partial^{2} w}{\partial \xi^{2}}+\frac{2 d \nu^{2} \partial w}{\nu_{0}^{2} \epsilon_{0} \partial \tau}+\frac{\nu^{2}}{\nu_{0}^{2} \epsilon_{0}} \frac{\partial^{2} w}{\partial \tau^{2}}=0 .
$$

3. The column with pinned ends. We concentrate now on the task of determining criteria associated with stable (bounded) solutions of the partial differential equation (2.4) together with the boundary conditions $w(\mathbf{0}, \boldsymbol{\tau})=w(\boldsymbol{\pi}, \boldsymbol{\tau})=w_{\xi \xi}(\mathbf{0}, \boldsymbol{\tau})=w_{\xi \xi}(\boldsymbol{\pi}, \boldsymbol{\tau})=0$.

We assume a solution, $w(\xi, \tau)$, of Eq. (2.4) in the form

$$
w(\xi, \tau)=\sum_{n=1}^{\infty} y_{n}(\tau) \sin n \xi,
$$

where the functions $y_{n}$ are to be determined. We note that a necessary condition that $w(\xi, \tau)$ be bounded is that each of the functions $y_{n}$ should be bounded for all $\tau$. 
If we assume that differentiation of the series can be performed, it is clear that $w$ as given in (3.1) satisfies the boundary conditions and satisfies Eq. (2.4) provided that

$$
\sum_{n=1}^{\infty}\left\{\frac{\nu^{2}}{\nu_{0} \epsilon_{0}} y_{n}{ }^{\prime \prime}(\tau)+\frac{2 d \nu^{2}}{\nu_{0}^{2} \epsilon_{0}} y_{n}{ }^{\prime}(t)+\left[n^{4}+\frac{n^{2}}{P_{E}} F\left(\frac{p}{2 \pi} \tau\right)\right] y_{n}(\tau)\right\} \sin n \xi=0 .
$$

We define $\beta_{n}$ and $\alpha_{n}$ by the relations

$$
\beta_{n}=\frac{n^{2} \nu_{0}^{2} \epsilon_{0}}{\nu^{2}}\left(n^{2}+\frac{\mu}{P_{E}}\right), \quad n=1,2,3, \cdots,
$$

and

$$
\alpha_{n}=\frac{n^{2} \nu_{0}{ }^{2} \epsilon_{0} 2 \pi \eta}{\nu P_{E}}, \quad n=1,2,3, \cdots .
$$

Thus condition (3.2) gives rise to an infinite sequence of ordinary differential equations, each one differing from any other in the sequence merely in the parameters involved. It suffices, then, to investigate the single equation

$$
y^{\prime \prime}(\tau)+2 d y^{\prime}(\tau)+\left[\beta+\alpha \delta_{2 \pi}(\tau)\right] y(\tau)=0
$$

for bounded solutions. In order to do this we make use of the techniques associated with the Floquet theory (see [2, pp. 193-204]).

4. Fundamental solutions, stability conditions, and the stability diagram. It suffices to investigate Eq. (3.5) on an interval of length $2 \pi$ which we take to be the interval $(-\pi / 2,3 \pi / 2) . y_{1}(\tau), y_{2}(\tau)$ denotes a fundamental set of solutions of Eq. (3.5) on $(-\pi / 2$, $\pi / 2)$, and $y_{3}(\tau), y_{4}(\tau)$ denotes a fundamental set on $(\pi / 2,3 \pi / 2)$. Matching the limits of the function $y$ and its first derivative $y^{\prime}$ at the point $\tau=\pi / 2$, we obtain the following connection formulae between the integration constants:

$$
\begin{aligned}
& c_{3}=\frac{1}{a_{34}}\left[a_{14} c_{1}+a_{24} c_{2}\right], \\
& c_{4}=\frac{1}{a_{34}}\left[a_{31} c_{1}+a_{32} c_{2}\right],
\end{aligned}
$$

where $a_{i i} \equiv W\left(y_{i}, y_{i}\right)(\pi / 2)$, i.e. the Wronskian of $y_{i}$ and $y_{i}$ evaluated at $\tau=\pi / 2$. For the study of boundedness of solutions, it is desired to find solutions such that

$$
y(3 \pi / 2)=\nabla y(-\pi / 2), \quad y^{\prime}(3 \pi / 2)=\nabla y^{\prime}(-\pi / 2),
$$

with $|\nabla| \leq 1$. Eqs. (4.3) give rise to the following linear algebraic system for $c_{1}$ and $c_{2}$ :

$$
\sum_{i=1}^{2} \gamma_{i j} c_{i}=0, \quad i=1,2
$$

with

$$
\gamma_{i i}=\frac{a_{i 4}}{a_{34}} y_{3}{ }^{(i-1)}\left(\frac{3 \pi}{2}\right)+\frac{a_{3 j}}{a_{34}} y_{4}{ }^{(i-1)}\left(\frac{3 \pi}{2}\right)-\nabla y_{i}{ }^{(i-1)}\left(-\frac{\pi}{2}\right) .
$$

The superscript $(i-1)$ in (4.4) denotes differentiation. Thus, we have nontrivial solutions for $c_{1}$ and $c_{2}$ if and only if 


$$
M \nabla^{2}+N \nabla+P=0,
$$

where $M=W\left(y_{1}, y_{2}\right)(-\pi / 2), N=\left(1 / a_{34}\right)\left[a_{14} b_{23}+a_{31} b_{24}-a_{24} b_{13}-a_{32} b_{14}\right]$,

$$
P=\frac{1}{\left(a_{34}\right)^{2}}\left(a_{14} a_{32}-a_{31} a_{24}\right) W\left(y_{3}, y_{4}\right)\left(\frac{3 \pi}{2}\right),
$$

with

$$
b_{i j} \equiv y_{i}\left(-\frac{\pi}{2}\right) y_{i}{ }^{\prime}\left(\frac{3 \pi}{2}\right)-y_{i}{ }^{\prime}\left(-\frac{\pi}{2}\right) y_{i}\left(\frac{3 \pi}{2}\right) .
$$

Utilizing standard theorems from the theory of ordinary differential equations $[1$, p. 87] and standard properties of delta functions, we have the following results:

$$
\begin{aligned}
& y_{1}(\tau)=\exp [-d \tau] v_{1}(\tau), \\
& y_{2}(\tau)=\exp [-d \tau]\left\{v_{2}(\tau)-\frac{\alpha}{2 \kappa} v_{1}(|\tau|)\right\}, \\
& y_{3}(\tau)=\exp [-d(\tau-\pi)] v_{1}(\tau-\pi), \\
& y_{4}(\tau)=\exp \left[-d(\tau-\pi)\left\{v_{2}(\tau-\pi)+\frac{\alpha}{2 \kappa} v_{1}(|\tau-\pi|)\right\},\right.
\end{aligned}
$$

where

$$
\begin{aligned}
v_{1}(\tau) & =\sin \left(\beta-d^{2}\right)^{1 / 2} \tau, & & \beta>d^{2} \\
& =\tau, & & \beta=d^{2} \\
& =\sinh \left(d^{2}-\beta\right)^{1 / 2} \tau & & \beta<d^{2}, \\
v_{2}(\tau) & =\cos \left(\beta-d^{2}\right)^{1 / 2} \tau, & & \beta>d^{2} \\
& =1, & & \beta=d^{2} \\
& =\cosh \left(d^{2}-\beta\right)^{1 / 2} \tau, & & \beta<d^{2},
\end{aligned}
$$

and

$$
\begin{aligned}
\kappa & =\left(\beta-d^{2}\right)^{1 / 2}, & & \beta>d^{2}, \\
& =1, & & \beta=d^{2}, \\
& =\left(d^{2}-\beta\right)^{1 / 2}, & & \beta<d .
\end{aligned}
$$

Furthermore, Eq. (4.5), after simplification, has the form

$$
\nabla^{2}-2 \exp (-2 \pi d) A \nabla+\exp (-4 \pi d)=0
$$

where

$$
A=v_{2}(2 \pi)-\frac{1}{2}\left[\frac{\alpha v_{1}(\pi)}{\kappa}\right]^{2} .
$$

From Eq. (4.6) we have

$$
\nabla_{+,-}=\exp (-2 \pi d)\left\{A \pm\left(A^{2}-1\right)^{1 / 2}\right\} .
$$

It is easy to see that all solutions are bounded when $A^{2} \leq 1$. 
When $\beta>d^{2}$, it is clear that $A$ can never exceed 1 ; in fact, $A=1$ only if $\beta=n^{2}+d^{2}$, $n$ an integer. The case $A<-1$ gives rise to the fact that both $\nabla_{+}$and $\nabla_{-}$are negative and that $\left|\nabla_{-}\right|>\left|\nabla_{+}\right|$; thus for boundedness it suffices to demand that $\operatorname{Re}\left(1 / 2 \pi \log \nabla_{-}\right)$ $\leq 0$, i.e.

$$
\alpha^{2} \leq 4\left(\beta-d^{2}\right)\left\{\cot ^{2} \pi \kappa+\sinh ^{2} \pi \csc ^{2} \pi \kappa\right\} .
$$

The equality in condition (4.8) yields the boundary curves of our stability diagram (for $\beta>d^{2}$ ). These curves are symmetrical with respect to the $\beta$-axis, have vertical asymptotes at $\beta=n^{2}+d^{2}, n$ a positive integer, and have relative extrema at the points $\left(\beta_{m}, \pm \alpha_{m}\right)$ where $\beta_{m}, m=0,1,2, \cdots$, is the root of the equation $\left(\kappa=\left(\beta-d^{2}\right)^{1 / 2}\right)$

$$
\left\{\cos ^{2} \pi \kappa+\sinh ^{2} \pi d\right\} \sin \pi \kappa-\pi \kappa \cosh ^{2} \pi \cos \pi \kappa=0,
$$

and $\alpha_{m}$ is obtained from the equality in (4.8), i.e. $\left(\kappa_{m}=\left(\beta_{m}-d^{2}\right)^{1 / 2}\right)$ :

$$
{\alpha_{m}}^{2}=4 \pi \kappa_{m}^{3} \cosh ^{2} \pi d \cot \pi \kappa_{m} \csc ^{2} \pi \kappa_{m} .
$$

It is easy to see that for all values of $d$ we have

$$
m^{2}+d^{2}<\beta_{m}<\left(\frac{2 m+1}{2}\right)^{2}+d^{2}, \quad m=1,2,3, \cdots .
$$

As for $\beta_{0}$ we have the following five cases (in cases (iii)-(v) the symbol $x_{*}$ represents the first root of the equation $x=\cot x$, i.e. $\left.x_{*} \simeq 0.86033\right)$ :

(i) if $d>(1 / \pi) \sinh ^{-1}(\sqrt{ } 2), \beta_{0}$ is non-existent;

(ii) if $d=(1 / \pi) \sinh ^{-1}(\sqrt{ } 2), \beta_{0}=d^{2}$;

(iii) if $\frac{1}{\pi} \sinh ^{-1}\left\{\left[\frac{x_{*} \cos x_{*}-\cos ^{2} x_{*} \sin x_{*}}{\sin x_{*}-x_{*} \cos x_{*}}\right]^{1 / 2}\right\} \equiv d_{1}<d<\frac{1}{\pi} \sinh ^{-1}(\sqrt{ } 2)$,

$$
d^{2}<\beta_{0}<\left(x_{*} / \pi\right)^{2}+d^{2}
$$

(iv) if $d=d_{1}, \beta_{0}=\left(x_{*} / \pi\right)^{2}+d^{2}$;

(v) if $0<d<d_{1},\left(x_{*} / \pi\right)^{2}+d^{2}<\beta_{0}<\frac{1}{4}+d^{2}$.

When $\beta=d^{2}$, the case $A>1$ is again impossible. The case $A<-1$ gives rise to the stability condition

$$
\alpha^{2} \leq\left(4 / \pi^{2}\right) \cosh ^{2} \pi d .
$$

When $\beta<d^{2}$ we have two subcases. In case $A>1$ the stability condition is $(\kappa=$ $\left.\left(d^{2}-\beta\right)^{1 / 2}\right)$

$$
\alpha^{2} \geq 4 \kappa^{2}\left\{\operatorname{coth}^{2} \pi \kappa-\cosh ^{2} \pi d \operatorname{csch}^{2} \pi \kappa\right\} .
$$

We note that if $0 \leq \beta<d^{2}$, condition (4.10) is always satisfied since its right-hand side is non-positive. When $A<-1$ the stability condition is given by

$$
\alpha^{2} \leq 4 \kappa^{2}\left\{\operatorname{coth}^{2} \pi \kappa+\sinh ^{2} \pi d \operatorname{csch}^{2} \pi \kappa\right\} .
$$

The equality in condition (4.11) yields a boundary curve in our stability diagram which is symmetrical with respect to the $\beta$-axis and has at most one minimum point $\left(\beta_{0}, \alpha_{0}\right)$ (in the upper half plane) where $\beta_{0}$ is a root of the equation $\left(\kappa=\left(d^{2}-\beta\right)^{1 / 2}\right)$

$$
\pi \kappa \cosh ^{2} \pi d \cosh \pi \kappa-\left\{\cosh ^{2} \pi \kappa+\sinh ^{2} \pi d\right\} \sinh \pi \kappa=0 .
$$


We have the following five cases:

(i) if $0<d<1 / \pi \sinh ^{-1}(\sqrt{ } 2), \beta_{0}$ is non-existent;

(ii) if $d=(1 / \pi) \sinh ^{-1}(\sqrt{ } 2), \beta_{0}=d^{2}$;

(iii) if $(1 / \pi) \sinh ^{-1}(\sqrt{ } 2)<d<d_{2}, 0<\beta_{0}<d^{2}$;

(iv) if $d=d_{2}, \beta_{0}=0$;

(v) if $d>d_{2},-\infty<\beta_{0}<0$,

where $d_{2}$ is the positive root of the equation $\pi d \cosh ^{3} \pi d=\sinh \pi d \cosh 2 \pi d$. Thus, $0.57<d_{2}<0.58$.

On the basis of the above analysis we are able to construct the stability diagram(s) in Fig. 1. The shaded regions are the stable regions. The diagram presented is drawn for $d=0.2$ (i.e., $0<d<(1 / \pi) \sinh ^{-1}(\sqrt{ } 2)$ ). We remark that the asymptotes for the boundary curves in the case $\beta>d^{2}$ correspond to $A=+1$ and hence belong to the stable regions. Also, in this case no solutions of period $2 \pi$ (i.e. $\nabla=+1$ ) exist since this would require that $A=\cosh 2 \pi d$ which is impossible. Solutions of period $4 \pi$ (i.e. $\nabla=-1$ ) occur along the boundary curves.

Thus, with regard to necessary conditions for column stability, a triple of parameter values $(\mu, \eta, \nu)$ will be termed stable if all points of the sequence generated by this triple, $\left\{\left(\beta_{n}, \alpha_{n}\right)\right\}_{n=1}^{\infty}$ (ref. (3.3) and (3.4)), are stable points. That is to say, $\left(\beta_{n}, \alpha_{n}\right)$ lies in the shaded regions of Fig. 1 for all $n$.

Theorem 4.1. Let $\gamma$ be any positive number. If $d>0, \beta>d^{2}+\frac{1}{4}$ and $\alpha^{2}<$ $4\left(\beta-d^{2}+\gamma\right) \sinh ^{2} \pi d /(1+4 \gamma)$, then all solutions of Eq. (3.5) are stable.

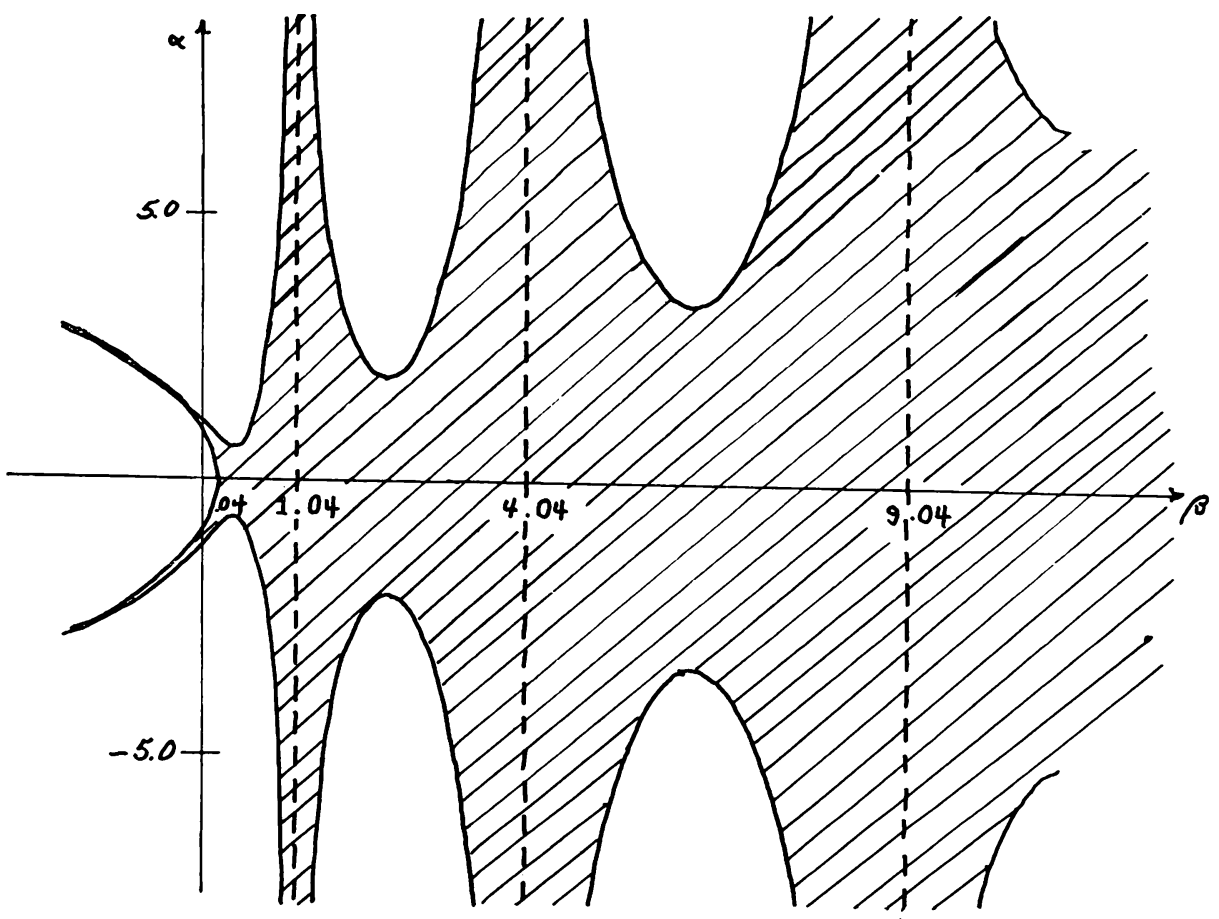

FIg. 1. Stability diagram for Eq. (3.5). 
We remark that a thoerem of the above type is impossible in the case of no damping (see Sec. 5).

Proof. Consider the intersections of the boundary curves of Fig. 1 in the case $\beta>d^{2}$ with the parabolas $\alpha^{2}=C\left(\beta-d^{2}\right)+B$. The abscissae of these intersections are roots of the equation

$$
4\left[\cos ^{2} \pi\left(\beta-d^{2}\right)^{1 / 2}+\sinh ^{2} \pi d\right]-\left(C+\frac{B}{\beta-d^{2}}\right) \sin ^{2} \pi\left(\beta-d^{2}\right)^{1 / 2}=0 .
$$

Clearly if $C+4 B<4 \sinh ^{2} \pi d$ then the above equation has at most roots in the interval $d^{2}<\beta<d^{2}+\frac{1}{4}$. Thus if we restrict $C$ and $B$ to satisfy $C+4 B<4 \sinh ^{2} \pi d$ and $\beta>$ $d^{2}+\frac{1}{4}$ the parabola $\alpha^{2}=C\left(\beta-d^{2}\right)+B$ lies entirely in the stable region. The theorem follows by setting $B=\gamma C$ where $\gamma$ is positive.

Q.E.D.

Thus, if $\left(\mu_{*}, \eta_{*}, \nu_{*}\right)$ is a triple of values for which the column is stable, then there exists a neighborhood of this triple consisting entirely of stable triples. Indeed, consider $\gamma$ as in Theorem 4.1 and assume $\nu$ to be arbitrary. Restrict $d$ to be such that $0<d^{2}<\gamma$, i.e. $h^{2}<16 \pi^{2} m^{2} \nu^{2} \gamma$. A set of necessary and sufficient conditions that $\alpha_{n}{ }^{2}<4\left(\beta_{n}-\right.$ $\left.d^{2}+\gamma\right) \sinh ^{2} \pi d /(1+4 \gamma)$ for all $n$ is as follows:

(i) $\eta^{2}<\frac{P_{E} \sinh ^{2} \pi d}{(1+4 \gamma) \pi^{2} \nu_{0}^{2} \epsilon_{0}}$,

$$
\mu^{2}<4 \nu^{2}\left[\frac{P_{E}^{2}}{\nu_{0}^{2} \epsilon_{0}}-4 \pi^{2} \eta^{2}\right]\left[\gamma-d^{2}\right] .
$$

5. No damping. If throughout the previous discussion one considers $\lim d \rightarrow 0^{+}$, one obtains an analysis of the undamped column [2]. In this section we present some of the main results. For a more complete discussion see [2].

As a first result we present the stability diagram in Fig. 2. It is worth noting that in this case the stability condition is $|A|<1$ ( $A$ being the limiting form of Eq. (4.7)) and the curves given by $|A|=1$ in general separate stable points from unstable points and themselves belong to the unstable regions. However, when $\beta>0$ the curves $A=+1$ are the lines $\beta=n^{2}, n$ an integer, and the solutions in this case are stable. Thus we have an example of the phenomenon referred to as coexistence [7].

5.1. Some necessary criteria for column stability. As before, a necessary condition that the undamped column with pinned ends be stable is that the applied force be such that all points $\left(\beta_{n}, \alpha_{n}\right)$ lie within the shaded regions of the stability diagram. In what follows we make the following simplifying substitutions:

$$
\gamma=-\mu / P_{E}, \quad \lambda=\nu / \nu_{0} \epsilon_{0}{ }^{1 / 2}, \quad \xi=\pi \nu_{0} \eta \epsilon_{0}{ }^{1 / 2} / P_{E} .
$$

In the paper by Lubkin and Stoker [5] one of the main interests was to show that a column made statically unstable by having $\mu<-P_{E}$, the Euler load, could be made dynamically stable by applying a suitably chosen additional oscillatory load.

The same results can be accomplished in this case [2]. We state here the main results. We restrict $\gamma$ to a range $1<\gamma<4$, because then the steady part of the applied force is a compression as much as four times greater than the Euler load.

Theorem 5.1.1. Let $\lambda=1$ and let $\epsilon$ be such that $\pi / 8<\cot ^{-1} \epsilon<\cot ^{-1}(2 / \sqrt{ } 3)$; then for all $\gamma$ such that $2-2 / \pi \cot ^{-1} \epsilon<\gamma<\frac{7}{4}$, and for all $\xi$ such that 


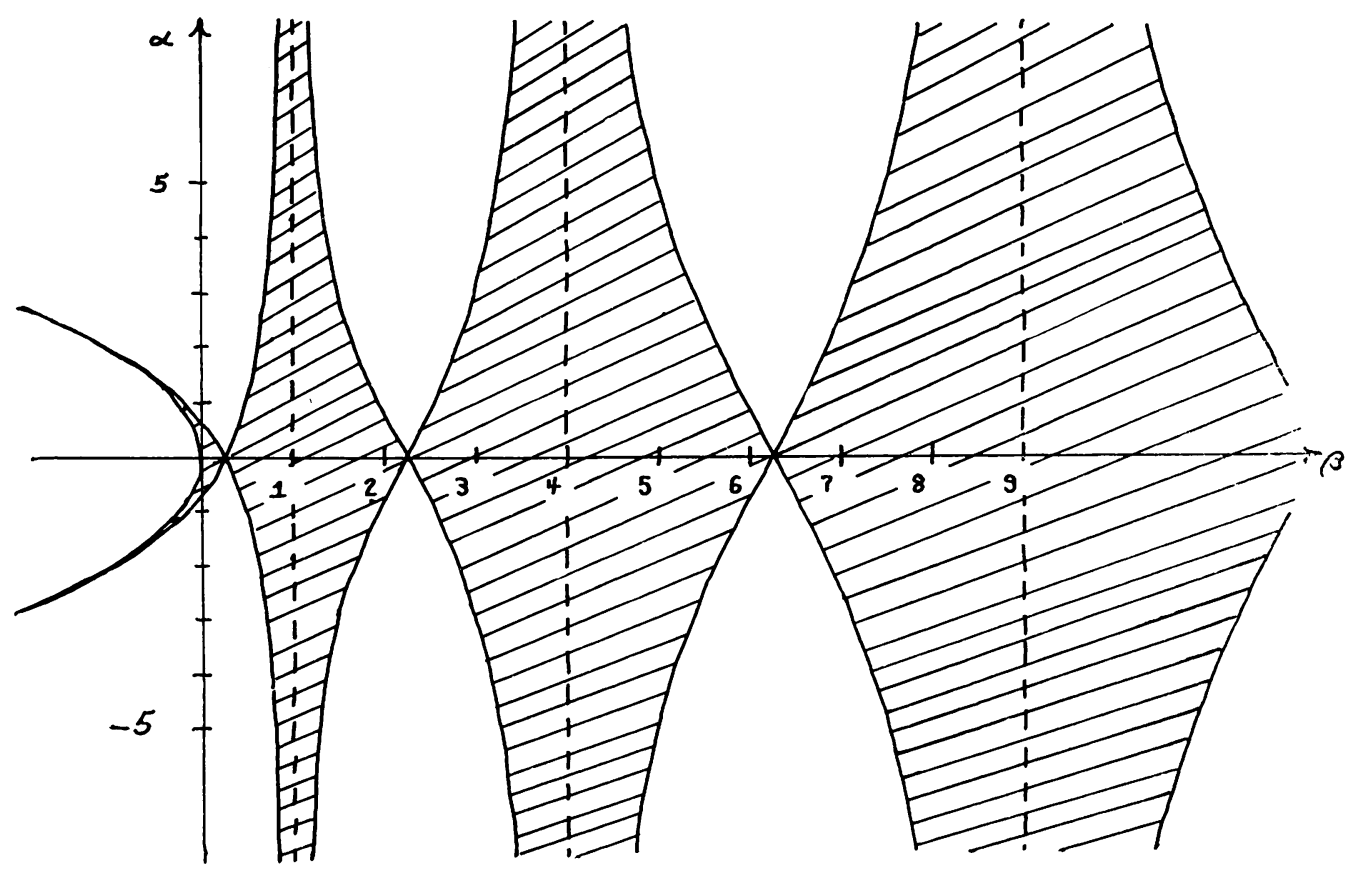

FIg. 2. Stability diagram for Eq. $(3.5)(d=0)$.

$$
\xi=b(\gamma-1)^{1 / 2}+(1-b)(\gamma-1)^{1 / 2} \operatorname{coth} \frac{\pi(\gamma-1)^{1 / 2}}{\lambda},
$$

where $b$ is such that

$$
\left(\operatorname{coth} \frac{\pi(\gamma-1)^{1 / 2}}{\gamma}-\frac{\epsilon}{2}\left(\frac{4-\gamma}{\gamma-1}\right)^{1 / 2}\right) /\left(\operatorname{coth} \frac{\pi(\gamma-1)^{1 / 2}}{\lambda}-1\right)<b<1,
$$

all points of the sequence $\left\{\left(\beta_{n}, \alpha_{n}\right)\right\}_{n=1}^{\infty}$ are stable points.

Theorem 5.1.2. Let $\lambda=2$ and let $\epsilon$ be such that $(2-\sqrt{ } 3) \pi<\cot ^{-1} \epsilon<\pi / 2$; then for all $\gamma$ such that

$$
1<\gamma<\min \left[\frac{4\left(\epsilon^{2}+1\right)}{4+\epsilon^{2}}, \frac{4\left(\cot ^{-1} \epsilon\right)}{\pi}-\frac{\left(\cot ^{-1} \epsilon\right)^{2}}{\pi^{2}}\right],
$$

and for all $\xi$ given by (5.1.2) and (5.1.3), we have that all points of the sequence $\left\{\left(\beta_{n}, \alpha_{n}\right)\right\}_{n=1}^{\infty}$ are stable points.

Theorem 5.1.3. Let $\lambda=4$ and let $\epsilon$ be such that $(4-\sqrt{ } 127 / 3) \pi<\cot ^{-1} \epsilon<\pi / 2$; then for all $\gamma$ such that

$$
1<\gamma<\min \left[\frac{4\left(\epsilon^{2}+1\right)}{4+\epsilon^{2}}, \frac{17}{9}\right],
$$

and for all $\xi$ given by (5.1.2) and (5.1.3), we have that all points of the sequence $\left\{\left(\beta_{n}, \alpha_{n}\right)\right\}_{n=1}^{\infty}$ are stable points.

The stable situations indicated by Theorems 5.1.1-5.1.3 are rather illusory from a physical point of view in that if $\left(\mu_{*}, \eta_{*}, \nu_{*}\right)$ is a stable triple then it is impossible to 
find a neighborhood of this triple consisting entirely of stable triples (as we did in the case of damping) [2].

5.2. A sufficient condition for stability. We assume that for each $n, y_{n}(\tau)$ is a bounded solution of Eq. (3.5) with $d=0$. Now (3.1) shows that $|w(\xi, \tau)| \leq \sum_{n=1}^{\infty}\left|y_{n}(\tau)\right|$. If we invoke the boundedness conditions (4.3), it is clear that a sufficient condition that $w(\xi, \tau)$ be bounded for all $\tau$ is that the series $\sum_{n=1}^{\infty}\left|y_{n}(\tau)\right|$ converge uniformly with respect to $\tau$ on the fundamental interval $(-(\pi / 2), 3 \pi / 2)$. We assume that $\beta_{n}$ is positive for all $n$. This assumption is not restrictive since from Eq. (3.3) we note that there exists an integer $N$ (depending on $\mu$ ) such that for all $n \geq N, \beta_{n}$ is positive.

Theorem 5.2.1. For each $n=1,2,3, \cdots$, let $y_{n}(\tau)$ be a bounded solution of Eq. (3.5) (with $d=0$ ). Let $\beta_{n} \neq m^{2}$ where $m$ is an integer. The series $\sum_{n=1}{ }^{\infty}\left|y_{n}(\tau)\right|$ is convergent for all $t \in(-(\pi / 2), \infty)$ if the series

$$
\sum_{1}^{\infty}\left[6\left|\cot \pi \sqrt{ } \beta_{n}\right|+4 \csc ^{2} \pi \sqrt{ } \beta_{n}\right]\left[\left|y_{n}\left(-\frac{\pi}{2}\right)\right|+\frac{1}{\sqrt{ } \beta_{n}}\left|y_{n}{ }^{\prime}\left(-\frac{\pi}{2}\right)\right|\right]
$$

is convergent. (For proof see [2].)

Remarks. (1) It is interesting to note that the form of our majorant series is similar to that in the paper of Lubkin and Stoker [5] where they had to use integral forms for the solutions in order to obtain convenient estimates. Here we benefit greatly from the fact that the solutions are known explicitly.

(2) The terms $y_{n}(-(\pi / 2))$ and $y_{n}^{\prime}(-(\pi / 2))$ of our majorant series are the Fourier (sine) coefficients, respectively, of the initial position $w(\xi,-(\pi / 2)) \equiv f(\xi)$ and the initial velocity $w_{\tau}(\xi,-(\pi / 2)) \equiv g(\xi)$, which naturally are assumed bounded. Thus it is not unreasonable to expect that the majorant series converges, particularly if the Fourier series for $f$ and $g$ are absolutely convergent. Roughly speaking, then, our theorem asserts that if the initial functions $f$ and $g$ are such that the series (5.2.1) converges, then $w$ is bounded for all time.

5.3 Comparison of the properties of Eq. (3.5) $(d=0)$ with known properties of Hill's equation. Hill's equation is conventionally defined in the literature (Magnus and Winkler [7]) as an ordinary differential equation of the form $y^{\prime \prime}+(\lambda+Q(\tau)) y=0$, where $\lambda$ is a real parameter and $Q(\tau)$ is a real continuous periodic function of period $\pi$ whose average value is zero. Thus it seems reasonable to refer to Eq. (3.5) (with $d=0$ ) as a discontinuous Hill's equation and compare its properties to those of Hill's equation.

In [2] there is presented a number of such comparisons, most of which are somewhat immediate observations. We restrict our attention here to the following result (Theorem 5.3.1).

In Fig. 2 we consider $\alpha$ as fixed, say $\alpha=B$; the intersections of this line with the parabola $\alpha^{2}=-4 \beta$ and with the boundary curves gives rise to a sequence $\left\{\beta_{m}\right\}_{m=1}^{\infty}$ having the property that if $\beta \in\left[\beta_{2 m}, \beta_{2 m+1}\right]$ then $(\beta, B)$ is an unstable point, whereas if $\beta \in\left(\beta_{2 m+1}, \beta_{2 m+2}\right)$ then $(\beta, B)$ is a stable point.

Known results concerning the lengths of the intervals of instability for Hill's equation [7, pp. 43, 66, 67] show that these lengths tend to zero as $\lambda$ tends to infinity. Our results are that these lengths, i.e. $\beta_{2 n+1}-\beta_{2 n} \equiv L_{n}$, tend to a nonzero constant. This contrast is not totally unexpected, since Meissner [8] and Hochstadt [3] showed that for particular discontinuous Hill's equations the lengths of the intervals of instability tend to infinity. The papers of Kronig and Penny [4] and Sommerfeld and Bethe [11] demonstrate 
examples of discontinuous Hill's equations for which these lengths tend to zero. It is this author's observation that the present example is the first to exhibit an intermediate result.

Theorem 5.3.1. For fixed $\alpha$, say $\alpha=B, \lim _{n \rightarrow \infty} L_{n}=2|B| / \pi$.

Proof. Due to the symmetry of the stability diagram with respect to the $\beta$-axis, there is no loss in generality by assuming $B>0$. Hence we consider the geometry of Fig. 3. From Fig. 3, it is clear that $P_{4}-P_{3}<P_{5}-P_{2} \equiv L_{n}<P_{6}-P_{1}$, i.e.

$$
[(n+1)-u(n+1)]^{2}-[n+u(n+1)]^{2}<L_{n}<[(n+1)-u(n)]^{2}-[n+u(n)]^{2},
$$

where $u(n)=(1 / \pi) \cot ^{-1}(B / 2 n)$ and $\cot ^{-1}$ denotes the principal value. Thus,

$$
(2 n+1)(1-2 u(n+1))<L_{n}<(2 n+1)(1-2 u(n)) .
$$

An application of l'Hospital's rule on both sides of (5.3.1) establishes the asserted limit.

Q.E.D.

6. Analysis of the solutions to the basic ordinary differential equations. Many of the specific Hill's equations that have been studied, in particular Mathieu's equation, do not lend themselves to explicit solution. Nevertheless, investigators (e.g. [6], [13]) have been able to discuss asymptotic (with respect to large values of the independent variable) forms of the various solutions. Also, in the case of Mathieu's equation, Young [15] has investigated the behavior of the solution with respect to the parameters in the differential equation.

Such investigations can be conducted with relative ease in the present case because the solutions are known explicitly. The results, however, are quite lengthy in form and hence we content ourselves by referring the interested reader to [2], where an extensive and rather complete treatment can be found.

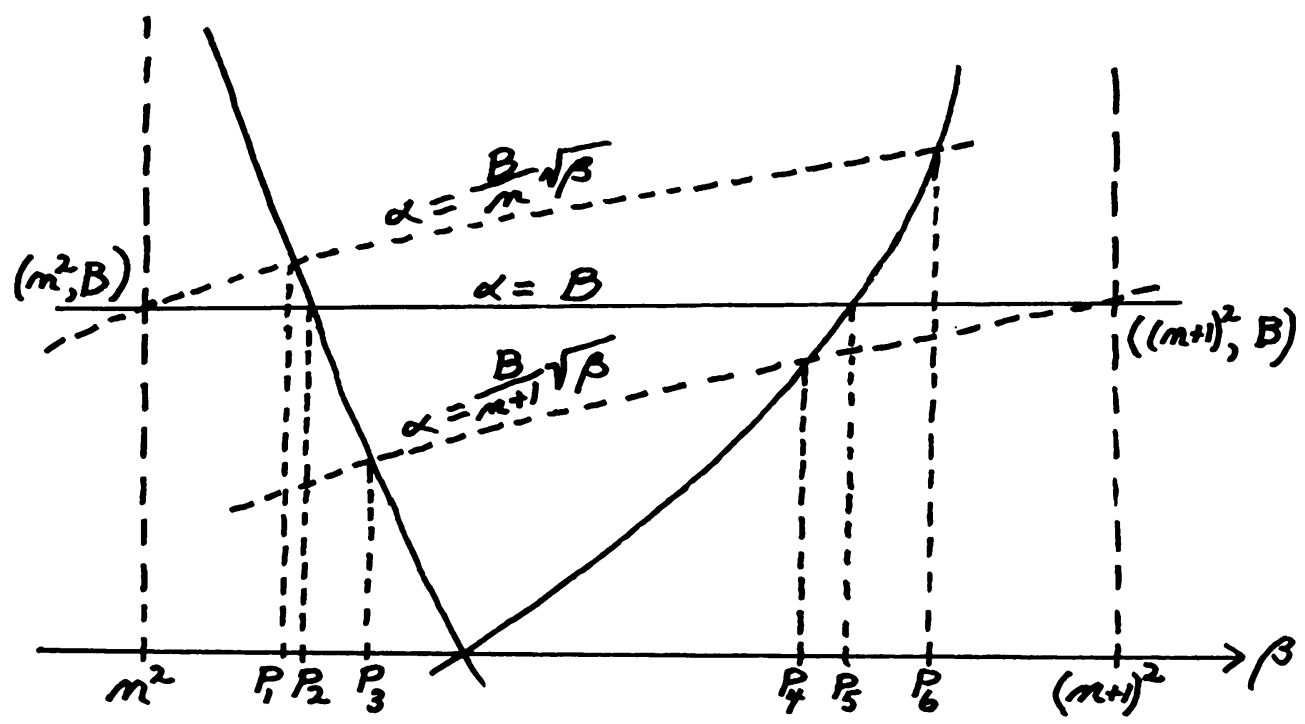

FIg. 3. 


\section{REFERENCES}

[1] E. A. Coddington and N. Levinson, Theory of ordinary differential equations, McGraw-Hill, New York (1955)

[2] N. J. Finizio, Two classical problems simplified by the introduction of periodic impulsive external forces, Ph.D. dissertation, New York University, 1972

[3] H. Hochstadt, A special Hill's equation with discontinuous coefficients, NYU Inst. Math. Sci., Div. of EM Res., Report BR-32 (1960)

[4] R. de L. Kronig and W. G. Penny, Quantum mechanics in crystal lattices, Proc. Royal Soc. London, 130, 215-236 (1918)

[5] S. Lubkin and J. J. Stoker, Stability of columns and strings under periodically varying forces, Quart. Appl. Math. 1, (1943)

[6] N. W. McLachlan, Theory and application of Mathieu functions, Oxford University Press, London (1951)

[7] W. Magnus and S. Winkler, Hill's equation, Interscience, New York (1966)

[8] E. Meissner, Uber Schuettelschwingungen in Systemen mit periodisch veraenderlicher Elastizitaet, Schweizer Bauzeitung, 72, No. 10 (1918)

[9] L. A. Pipes, Matrix solutions of equations of the Mathieu-Hill type, J. App. Phys. 24 (1953)

[10] L. A. Pipes, The dynamic stability of a uniform straight column excited by a pulsating load, J. Franklin Inst. 277 (1964)

[11] A. Sommerfeld and H. Bethe, Elektronen Theorie der Metalle, in Handbuch der Physik, second ed., Vol. 24II (1933)

[12] J. J. Stoker, Nonlinear vibrations in mechanical and electrical systems, Interscience, New York (1950)

[13] M. J. O. Strutt, Lamesche Mathieusche und Verwandte Functionen, Ergebnisse der Math. 3, Springer, Berlin (1932)

[14] S. Timoshenko, Vibration problems in engineering, Van Nostrand, Princeton (1928)

[15] A. W. Young, Quasi-periodic solutions of Mathieu's equation, Proc. Edinburgh Math. Soc. 32 (1914) 